\title{
On the Efficient Computation of Near-Field Radar Cross Sections
}

\author{
Emmanuel Van Lil, Jan-willem De Bleser \\ Div. ESAT-TELEMIC \\ KU Leuven \\ Kasteelpark Arenberg, 10; Bus 2444; B-3001 Heverlee, Belgium \\ Emmanuel.VanLil@ESAT.KULeuven.Be
}

\begin{abstract}
In some cases the objects like wind turbines are so large that the far-field condition are not met within the visibility of the object due to the curvature of the earth. Therefor, new ways to compute the reflections of those objects are required. This is similar to the concept of near-field communications, where the far-field gain (the normal definition) has to be replaced by the near-field gain. In some cases, we will even be able to find simple formulas for the worst case RCS in function of the relative size of the object with respect to the wavelength.
\end{abstract}

\section{Index Terms-RCS; near-field; wind turbine;}

\section{INTRODUCTION}

Even if the RCS bible from Ruck e.a. [1] contains a lot of RCS data, both monostatic and bistatic as well as a comprehensive formulation of the phenomena involved in the classical high frequency approximations (amongst others creeping waves), a full wave analysis was performed and compared with other approximations like PO.

First the electromagnetic simulation will be detailed (section II), then some examples for simple shapes will be given in view of previously commented simple approximations. (section III). This will inspire us for a discussion on the choice of the meshes to increase accuracy. Conclusions will be drawn in section V.

\section{ELECTROMAGNETIC SIMULATION}

A low frequency stabilized Moment methods based solution was developed previously [2]. It uses a combined charge and current formulation of which the charge could be eliminated, leading to a very efficient formulation for both dielectric ans PEC objects.

$$
\left[\begin{array}{l}
E_{i n c} \\
H_{i n c}
\end{array}\right]=\left[\begin{array}{cc}
\frac{c_{2}^{2} M_{1}^{r}-\kappa c_{1}^{2} M_{2}^{r}}{\varepsilon_{1} c_{1}^{2} c_{2}^{2}} & M_{1}^{c}-\frac{\varepsilon_{2} \kappa M_{2}^{c}}{\varepsilon_{1}} \\
M_{1}^{c}-\frac{\mu_{2} \kappa M_{2}^{c}}{\mu_{1}} & \frac{-c_{2}^{2} M_{1}^{r}+\kappa c_{1}^{2} M_{2}^{r}}{\mu_{1} c_{1}^{2} c_{2}^{2}}
\end{array}\right]\left[\begin{array}{l}
J_{s, e} \\
J_{s, \mathrm{~m}}
\end{array}\right]
$$

with $\kappa=M_{1}^{b}\left(M_{1}^{b T} M_{1}^{b}\right)^{-1} M_{1}^{b T}$

Note that both normal and tangential components of the incident field can be used, leading to a high accuracy. For the case of a sphere, of course, the analytical solution exists as a series of spherical orthogonal functions and was found by Mie [3]. In Fig. 1 we can indeed see that the accuracy of the combined charge and current formulation is best, even in the monostatic case the difference is very small.

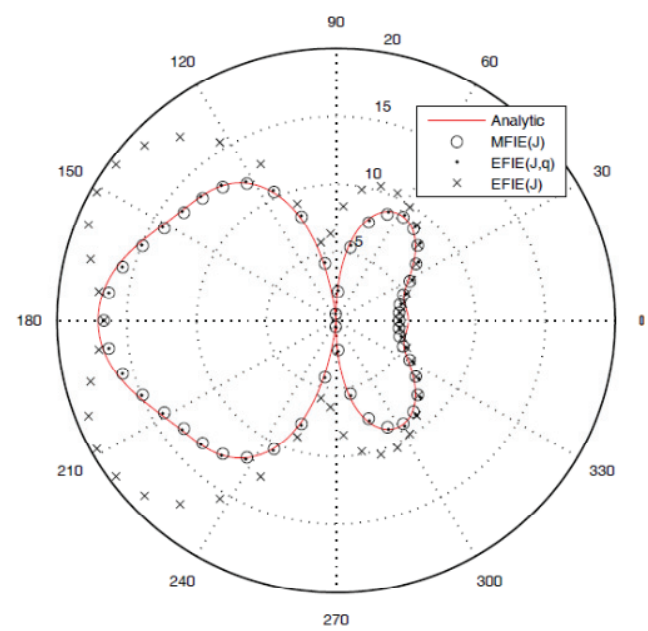

Fig. 1: RCS computations for an $r=\lambda / 2$ PEC sphere with 3 kinds of integral equation implementations.

One should also notice that in the limit case for small spheres the monostatic RCS is proportional to $9 \pi \mathrm{a}^{2}(\mathrm{ka})^{4}$, while a PO computation would give us the result $64 / 9$ or $7.111 \pi^{2}(\mathrm{ka})^{4}$, which is found on many web sites, even if the slope of the curve is similar. We should not wonder about this difference, since PO is only valid for surfaces that are nearly flat, which is not the case for a small sphere. The PO completely fails for large sphers as can be seen in . Another PO approximation, using only the illumnated part of the sphere, obviously collapse for small spheres, since this assumption is only valid is the sphere is much larger that the wavelength. 


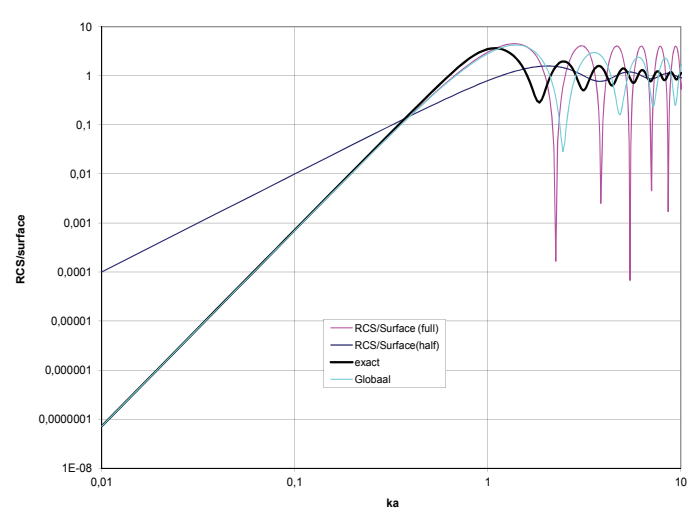

Fig. 2: PO RCS computations for a PEC sphere in function of its size and the exact value.

It is even remarkable that the difference is so small! For RCS computations at one frequency and different distances, we can easily solve (1) with many incident fields, corresponding with the different distances from the source to the object, making the solution even more efficient, since the matrices in the righ hand side remain the same.

\section{EXAMPLES}

Let us now look at the case of the monostatic RCS of a PEC sphere normalized with respect to its physical crosssection $\pi \mathrm{a}^{2}$ (Fig. 3).

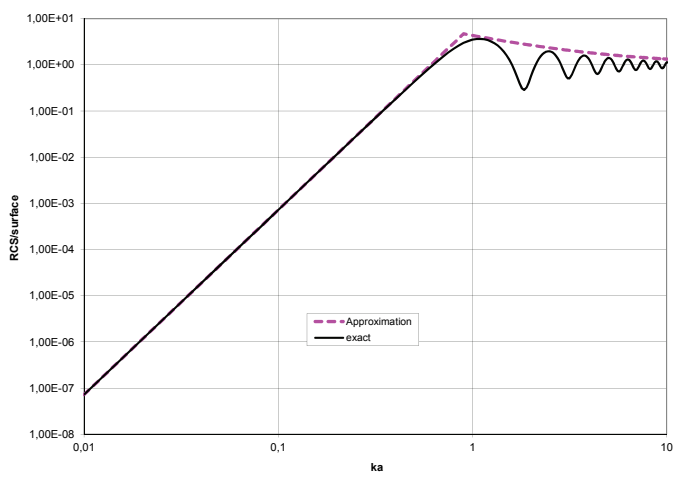

Fig. 3: Normalized monostatic RCS of a PEC sphere.

The most simple approximation would be to use a straight line equal to 1 for $\mathrm{ka} \geq 0.577$. This is similar to the approximation for the Fresnel functions used in the ITU-R P526 approximation for the loss of a semi-infinite screen. However, this would underestimate the real RCS for $\mathrm{ka} \approx 1$ by up to a factor 4.3 or more than $3 \mathrm{~dB}$. We should then use this as a safety factor for computations, increasing the safety distance for a radar with over $40 \%$. Fortunately, one can easily show that the envelope of the oscillations for ka $>1$ decays inversely proportionally with ka. The dotted line, representing $1+3.339 / \mathrm{ka}$ is an extremely good worst case approximation for the real RCS of the sphere. This can be used in case some regulatory bodies require the worst case reflections to guarantee that the interference will stay below a certain level.

A similar figure for the monostatic RCS of a finite PEC cylinder with dimensions of $10 \mathrm{~m}$ in height and $1 \mathrm{~m}$ radius is shown for a wavelength of $1 \mathrm{~m}$ in Fig. 4. The polarization of the transmitting dipole is parallel with the axis of the cylinder.

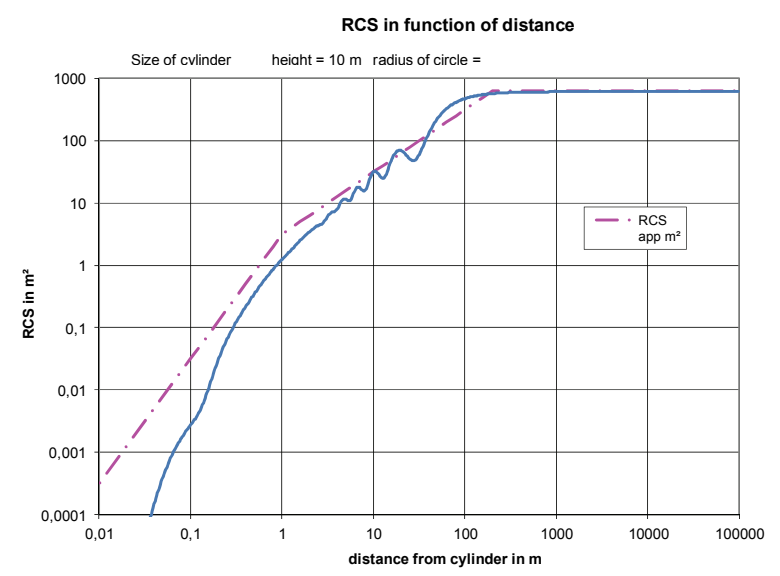

Fig. 4: Normalized monostatic near-field RCS of a PEC cylinder with a proportion $h / r=5$ and $r=\lambda$ in function of the distance to the specular reflection point.

We had already shown the linear approximation for the cylinder in [5], represented here in the dash-dotted line. Now we can see that only in a few points this value is exceed by up to $66 \%$ or $2 \mathrm{~dB}$. The far-field value of $2 \pi r h^{2} / \lambda$ or in this case $200 \pi$ is well followed after the last breakpoint at $200 \mathrm{~m}$. Below the first breakpoint at $1 \mathrm{~m}$, the approximation is not so well followed, but the approximation is always larger than the (already very small) real RCS. Also, this distance is more of academic value, since in practice no radar or target should be so close to the interfering object. We note however a relatively large relative error when the transmitter is very close to the object. This is indeed a difficult problem to solve. As we have previously experienced in the case of PO, the mesh should be adapted to the presence of the source and cannot be considered alone any more. This will force us to discuss the influence of the meshing both for expansion as for testing functions.

\section{ACCURACY CONSIDERATIONS}

We have already shown that using a combination of tangential and normal boundary conditions was increasing the accuracy. This forced us to end up with non-square MoM matrices. We will now prove for a simple case that this is not at all an inefficient idea. We will even simplify the problem further, and consider the case of a static 2D strip, already solved by Maxwell over 100 years ago. We first consider the case where the number of (pulse) expansion function is equal to the number of testing functions (point matching). The normalized charge distribution is shown in Fig. 5. 


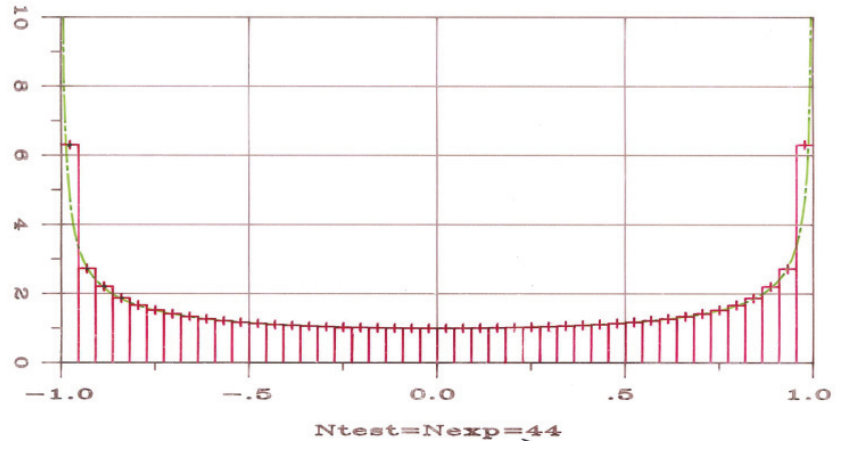

Fig. 5: Normalized charge distribution on a static strip (center charge $=1 \mathrm{C} / \mathrm{m}^{2}, 44$ equally spaced test and expansion functions).

It is obvious that, when we increase the number of test and expansion functions, the accuracy with which the exact distribution (becoming infinite at the edge of the strip) is followed increases (Fig. 6 for $\mathrm{N}=1250$ ).

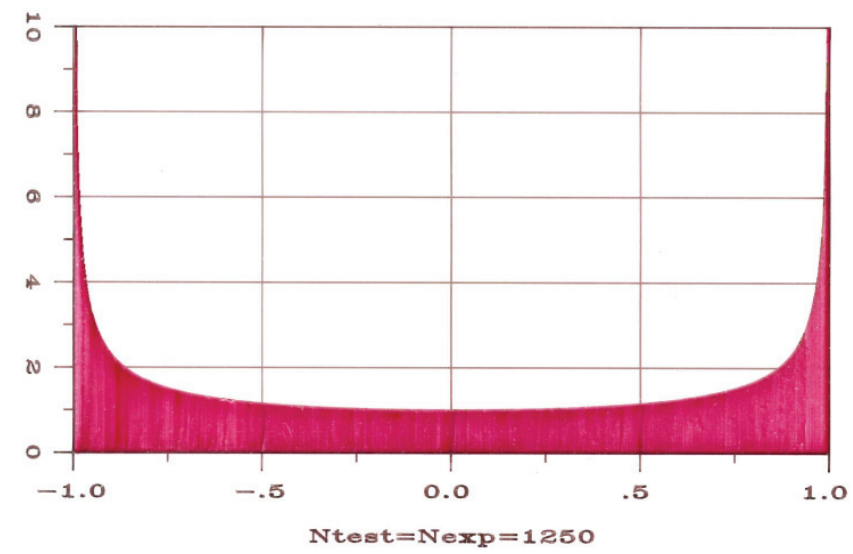

Fig. 6: Normalized charge distribution on a static strip (center charge $=1 \mathrm{C} / \mathrm{m}^{2}, 1250$ equally spaced test and expansion functions; the exact solution (green dash-dotted line) is barely visible).

Since the matrix is in this case Toeplitz very efficient algorithms exist to do so. The computational effort only increases with $\mathrm{N}^{2}$, while the matrix only require $\mathrm{N}$ memory positions. Of course many accuracies can be defined. If one considers the edge function, the absolute error becomes larger and larger. The error near the center of the strip becomes smaller and smaller. We will consider here a global value, like the capacitance of the strip (this can be compared with the global RCS for the problem at hand). It is obvious that the error continuously decreases when the number of functions (or mesh elements) increase (Fig. 7). The rate of the relative accuracy is approximately $1 / \mathrm{N}^{2}$.

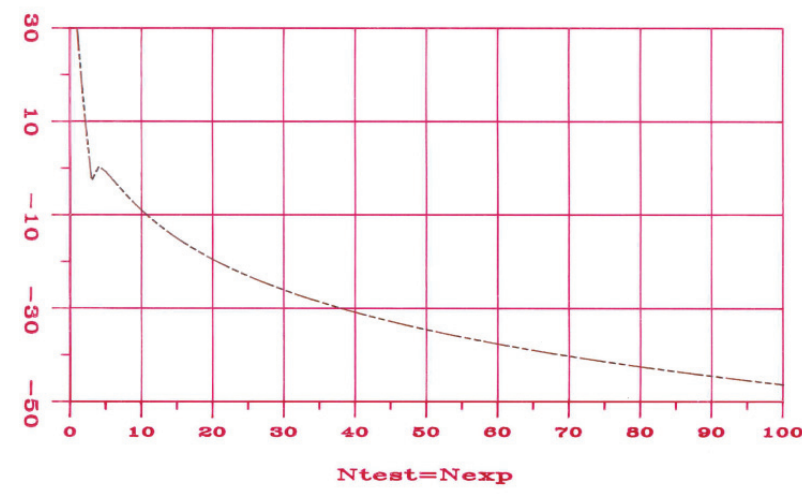

Fig. 7: Relative error on the capacitance in function of the number of equally spaced functions (logarithmic scale; $0=1 \%$, $-20=0.1 \%$ ).

It is also obvious, that, if we increase the of the mesh where the variable to be solved (in this case the charge density) varies the most, we will obtain a much better result with much less functions. For the strip case, the obvious choices are the zeroes of Chebyshev polynomials. The charge density with unequally spaced distributions is shown in Fig. 8.

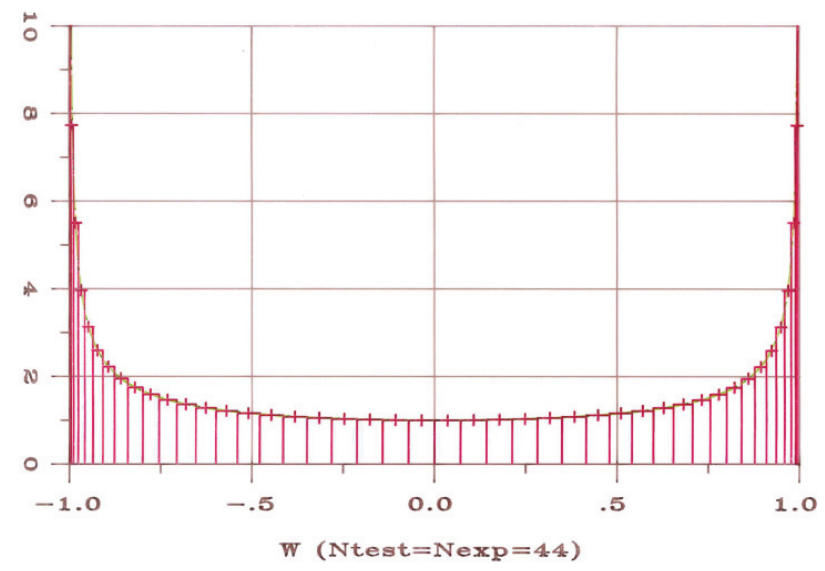

Fig. 8: Normalized charge distribution on a static strip (center charge $=1 \mathrm{C} / \mathrm{m}^{2}, 44$ unequally spaced test and expansion functions).

We can see that the capacitance error is now decreasing much more rapidly in function of the number of functions (Fig. 9). The decrease rate still remains $1 / \mathrm{N}^{2}$, but we will reach a high accuracy much faster. It should also be noted that the computational effort is now increased tot $\mathrm{N}^{3}$, since the matrix has no special features like Toeplitz, but this is a special case for geometries with a lot of symmetries that do not occur very often in practice. 


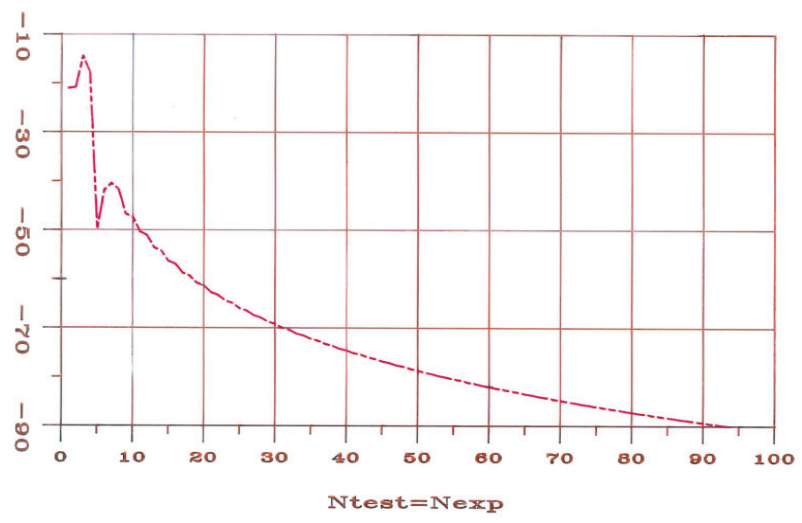

Fig. 9: Relative error on the capacitance in function of the number of unequally spaced functions (logarithmic scale; $0=$ $1 \%,-20=0.1 \%)$.

Finally, we can investigate the case of a different (larger) number of testing functions with respect to the number of expansion functions. One example of a charge distribution with 9 expansion functions and more testing functions is given in Fig. 10.

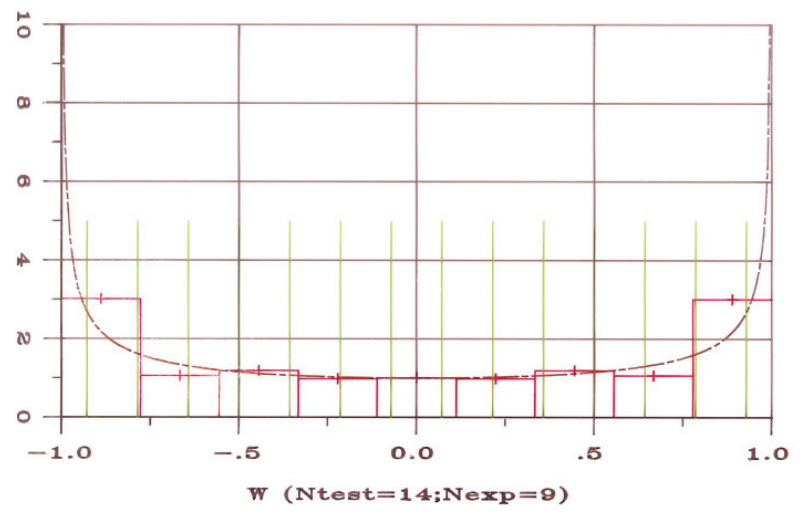

Fig. 10: Normalized charge distribution on a static strip (center charge $=1 \mathrm{C} / \mathrm{m}^{2}, 9$ equally spaced expansion and 14 equally spaced test functions (green Dirac impulses); the exact solution is shown in dark dash-dotted line).

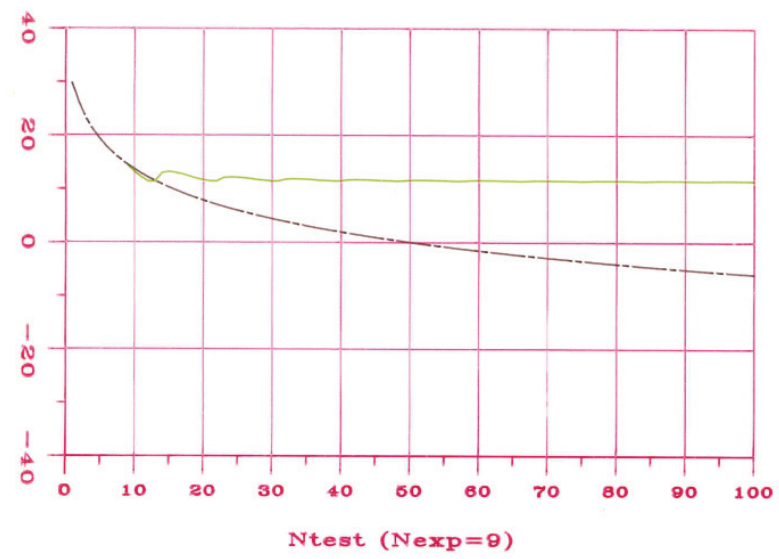

Fig. 11: Relative error on the capacitance in function of the number of equally spaced testing functions (logarithmic scale; 20 $=1 \%, 0=0.01 \%$; the number of expansion functions is 9 ).
Finally, we can see, that the accuracy increases, as long as we do not make the matrix too rectangular (Fig. 11). A further increase does not improve the accuracy.

The advantage of this procedure is that the computational time only increases linearly with the number of extra testing functions. This is performed on an old HP-1000 computer in with a real-time operating system (RTE6) (Fig. 12).

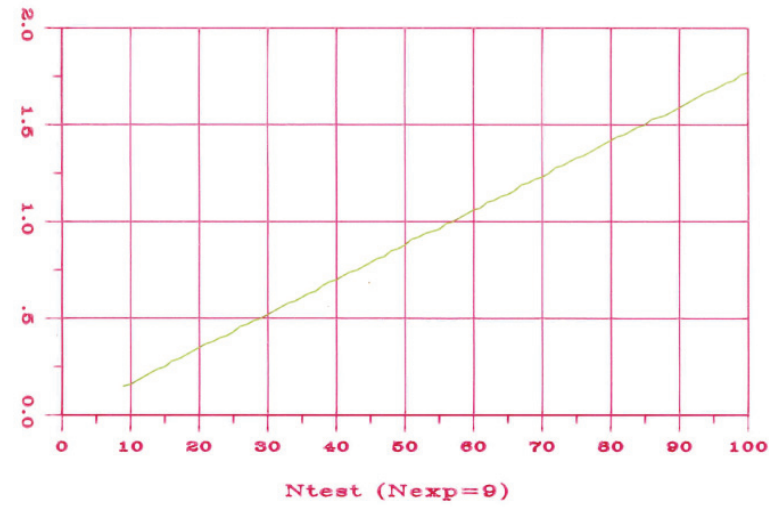

Fig. 12: Cpu time in functions of the number of test functions (9 expansion functions).

\section{CONCLUSION}

A useful formula which can be used in efficient programs has been derived for spheres. Some considerations about the choice of meshes to increase the full-wave solution accuracy have also been given. Other cases are more complex since they involve more parameters but are being investigated.

\section{REFERENCES}

[1] George T. Ruck, Donald E. Barrick, William D. Stuart and Clarence K Kirchbaum, "Radar Cross Section Handbook", Peninsula Publishing, 2002, ISBN-0-932146643

[2] Jan-willem De Bleser, Emmanuel H. Van Lil and Antoine R. Van de Capelle, "Split Formulation of the Charge and Current Integral Equations for Arbitrarily-Shaped Dielectrics", IEEE trans. Antennas and Propagat., Vol. AP-61, No. 1, pp. 302-310, January 2014

[3] G. Mie, "Beiträge zur Optik Truber Medien Speziell Kolloidaler Metallösungen", Annalen der Physik, vol. 25, pp. 377-445, 1908.

[4] ITU-R, Recommendation P-526, "Propagation by diffraction",

[5] Emmanuel H. Van Lil, Jan-willem De Bleser and Antoine R. Van de Capelle, " Computations of the Effects of Wind Turbines in the close near field of RF Installations", EUCAP 2011, pp. 1-5, Rome, 11-15 April 2011 